\title{
Gaze Manipulation for One-to-one Teleconferencing
}

\author{
A. Criminisi, J. Shotton, A. Blake, P.H.S. Torr \\ Microsoft Research Ltd, Cambridge, UK
}

\begin{abstract}
A new algorithm is proposed for novel view generation in one-toone teleconferencing applications. Given the video streams acquired by two cameras placed on either side of a computer monitor, the proposed algorithm synthesises images from a virtual camera in arbitrary position (typically located within the monitor) to facilitate eye contact.

Our technique is based on an improved, dynamicprogramming, stereo algorithm for efficient novel-view generation. The two main contributions of this paper are: i) a new type of three-plane graph for dense-stereo dynamic-programming, that encourages correct occlusion labeling; ii) a compact geometric derivation for novel-view synthesis by direct projection of the minimum-cost surface. Furthermore, this paper presents a novel algorithm for the temporal maintenance of a background model to enhance the rendering of occlusions and reduce temporal artefacts (flicker); and a cost aggregation algorithm that acts directly on our three-dimensional matching cost space.

Examples are given that demonstrate the robustness of the new algorithm to spatial and temporal artefacts for long stereo video streams. These include demonstrations of synthesis of cyclopean views of extended conversational sequences. We further demonstrate synthesis from a freely translating virtual camera.
\end{abstract}

\section{Introduction}

This paper addresses the problem of novel-view synthesis from a pair of rectified images with specific emphasis on peer-to-peer teleconferencing. With the rise of instant messenging technologies, it is envisaged that the PC will increasingly be used for interactive visual communication. One pressing problem is that any camera used to capture images of one of the participants has to be positioned offset to his or her gaze. This can lead to lack of eye contact and hence undesirable effects on the interaction [4].

Previously proposed solutions to this problem can be broadly categorized as model-based or image-based. One model-based approach is to use a detailed head model and reproject it into the cyclopean view; whilst this can be successful $[10,11]$, it is limited to imaging heads, and would not, for example, deal with a hand in front of the face or whiteboard scribbling. A more general approach therefore is to use low level stereo matching, in some form, and follow with an image based rendering approach (IBR) [1]. The
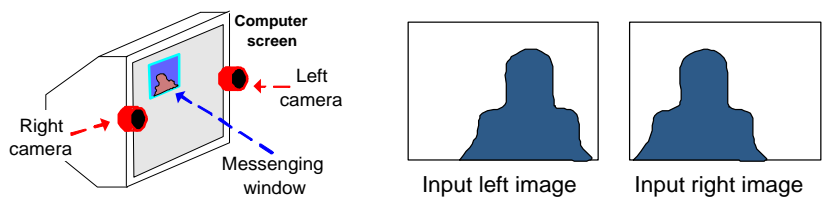

Figure 1: Camera configuration. The basic setup considers two cameras placed on the frame of the computer monitor. The goal of this paper is that of generating high-quality images for virtual cameras placed anywhere near the computer monitor. It will be demonstrated that gaze correction can be achieved by this technique in an efficient and compelling way.

aim is to synthesize a view from a virtual camera that is located roughly where the image of the head will be displayed on the screen for each participant, thus achieving eye contact. The basic setup is illustrated in fig. 1 .

In IBR a depth map is combined with an image to produce the new view. However in our approach we use a new min-cost surface projection algorithm for novel view generation that deals with occlusions and hole filling in a direct manner; by avoiding the explicit construction of the scene 3D model.

In order to generate a depth map a dense stereo algorithm is required, a substantial review of which can be found in [8]. According to the evaluation, two of the most powerful approaches use graph cuts $[5,7]$ and loopy belief propagation [9] but both of these are currently too computationally intensive for real time applications. Furthermore, the evaluation in [8] may not be valid for our purposes as: (i) the range of disparities considered in this paper is much smaller than in our application (0-29 pixels there, whereas we typically consider 0-80 pixel disparities), (ii) we are primarily interested in new view synthesis, thus it does not matter if the disparities are relatively inaccurate in homogeneous image regions, all that matters is that the new view is well synthesized. On the other hand where occlusions occur it is important to estimate them accurately since otherwise artefacts such as "haloing" (a lens-like distortion around foreground objects) become visible. (iii) we consider long video sequences, thus stability of estimation also plays a part: a flickery reconstruction is less desirable than a consistent one.

One of the most computationally efficient algorithms for stereo is Epipolar line Dynamic Programming [6], referred to as DP. We have implemented DP for dense stereo [3], 


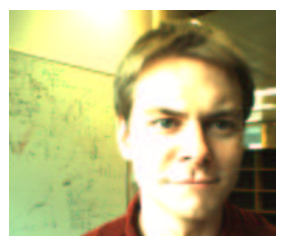

$\mathbf{a}$

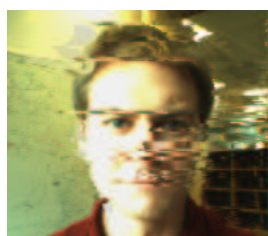

b

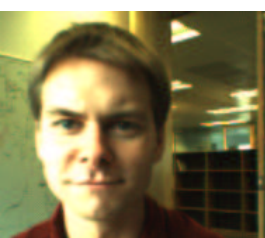

Figure 2: Fast cyclopean view synthesis by dynamic programming. (a,c) Input left and right views, respectively; (b) Cyclopean view synthesized by standard dynamic programming [2]. Note that gaze is correct in the cyclopean view. The algorithm runs at near real-time rate, but produces artefacts in the cyclopean image. In this case considerable undesired streaks corrupt the synthesized face. Furthermore, a reconstructed temporal cyclopean sequence shows considerable flicker and also an undesirable "halo" effect around the head.

the use of which has previously been demonstrated for cyclopean view interpolation [2] in video. To obtain computational efficiency, observations consist of single-pixel intensities, and the consequent quality of reconstruction (especially on cluttered backgrounds) is not consistently satisfactory, as fig. $2 b$ shows.

Reconstruction from standard dynamic programming is characterized by two kinds of error: (i) artefacts produced by mismatches (horizontal streaks), and (ii) the "halo" in the regions where the background is visible in only one of the two input views.

This paper sets out to address and solve both kinds of artefacts. There are two parts to our method: the first is generating accurate disparity and occlusion maps. Indeed, as will be seen, accurate labeling of occlusions is necessary to remove the "haloing" effect. The second is representing and using the computed disparities and occlusions to generate a new view. Within the paper we present new contributions in both areas: For the generation of disparities we propose a new type of dynamic programming approach, as path finding through a three-plane graph (as opposed to the traditional single-plane DP), introducing new labels to help the correct identification of occlusions, and altering the cost function employed to favour: (i) correct grouping of occlusions, (ii) formation of occlusions at the boundaries of foreground objects, and (iii) inter scanline consistency. Second, we introduce the elegant geometry of min-cost surface projection as an efficient technique for generating synthetic views from arbitrary virtual cameras directly from the minimum-cost surface obtained during the DP process.

The new algorithm is demonstrated on long sequences of pairs of synchronized stereo videos taken from static cameras, the methods show compelling novel view generation results.

Section 2 describes traditional dynamic programming. Section 3 introduces our improved dense stereo technique. Our view generation approach is described in section 4 , and finally, section 5 presents concluding results.

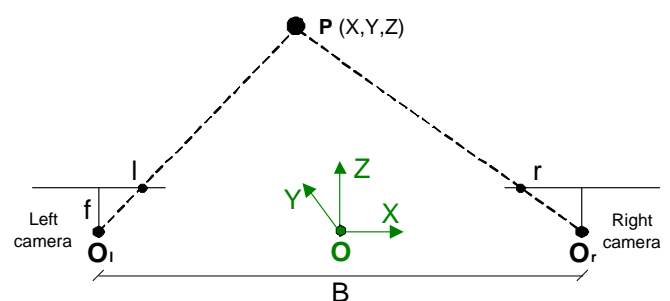

Figure 3: Basic notation. $\mathbf{P}$ is a 3D scene point. $\mathbf{O}_{l}$ and $\mathbf{O}_{r}$ are the optical centres of left and right cameras, respectively. $f$ is the focal length of the cameras and $B$ is the baseline between the two optical centres. $\mathbf{O}$ is the origin of the reference $X, Y, Z$ coordinate system.

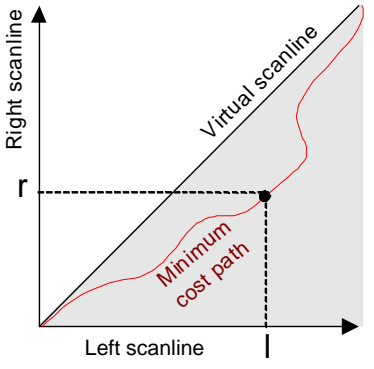

a

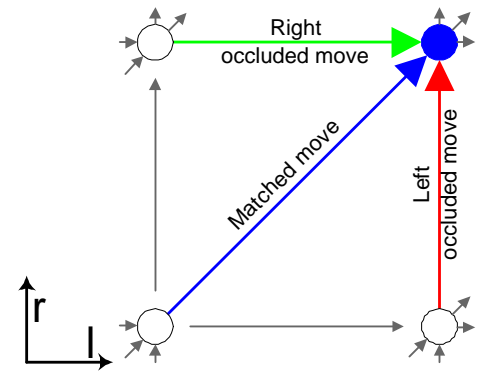

b
Figure 4: Traditional dynamic programming. (a) Basic diagram for dynamic programming. It represents the matrix of cumulative costs used for computing the minimum cost path. (b) A single-step view of (a) showing the set of the three allowed moves between pixel pairs in [2]. The circles represent elements of the cost matrix in (a).

\section{Traditional dynamic programming}

In the interests of clarity this section describes briefly the basic dynamic programming algorithm [3].

Figure 3 shows a plan view of the camera setup. The left and right cameras provide us with the synchronized and rectified input videos. $f$ is the focal length and $B$ the distance between the two optical centres (baseline). A Cartesian coordinate system is chosen with origin at the mid point between the left and right optical centres. In the remainder of the paper we will refer to cyclopean images as the ones generated by a virtual camera with optical centre in $\mathbf{O}$.

Given the configuration in fig. 3 the diagram in fig. $4 \mathrm{a}$ represents the cumulative cost matrix for a pair of corresponding scanlines in the two input images [3].

The cost $M(l, r)$ of matching a pixel at position $l$ along the left scanline and a pixel at position $r$ along the right scanline is defined simply as the square difference of pixel intensities (which in our experiment in fig. 2 is normalized between 0 and 1). Note that, since $l>=r \quad \forall \mathbf{P}$ (i.e. disparities are always positive), then it is only ever necessary to consider the lower half of the diagram (grey in fig. 4a). The limiting case $l=r$ corresponds to a point at infinity with consequent zero disparity. It is assumed from here on that 


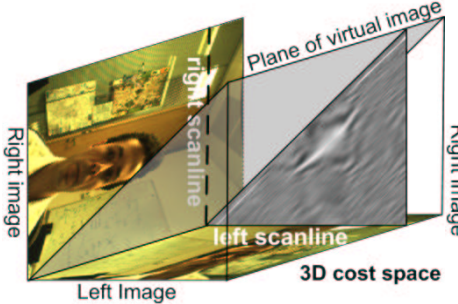

a

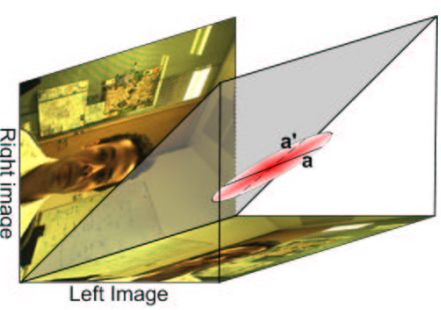

$\mathbf{b}$
Figure 5: The 3D cost space for a pair of stereo images. (a) The basic diagram of fig. $4 \mathrm{a}$ becomes a 3D diagram when all the scanline pairs are considered. The value of each element inside the parallelepiped is the $M(l, r)$ cost of matching two pixels. See text for details. The diagonal plane is called the "plane of virtual image" for reasons that will become apparent in the remainder of the paper. (b) A 2D Gaussian filter parallel to the virtual image plane is applied to the $3 \mathrm{D}$ cost space to enforce interscanline consistency.

any computation is restricted in this fashion.

Apart from a simple initialization step, the elements of the cumulative cost matrix $\mathrm{C}$ are filled, at each iteration by the following recurrence:

$$
\mathrm{C}(l, r)=\min \begin{cases}\mathrm{C}(l-1, r) & + \text { OccCost } \\ \mathrm{C}(l-1, r-1) & +M(l, r) \\ \mathrm{C}(l, r-1) & + \text { OccCost }\end{cases}
$$

where $\mathrm{C}(l, r)$ indicates the cumulative cost of the path reaching the point $(l, r)$ in the matrix. This recurrence defines the forward pass of the DP algorithm. Notice that three moves (or labels) are permitted: a horizontal occluded move, a diagonal matched move and a vertical occluded move, respectively.

The cost of having an occluded pixel, OccCost is a manually set parameter which depends on the image pair being examined - a value of 0.3 seems to yield the best results on a variety of images. At each iteration the minimum cost between the three possible moves is chosen and a table of backwards links is stored for use in the second part of DP. The backward pass of the algorithm follows the saved links, producing the minimum-cost path (fig. 4a) and, thereby the disparity map.

It is important to stress the fact that only three types of move are possible in [3] thus confounding occluded and non-occluded moves. As described later, one of the main contributions of this paper is that of expanding the set of permitted moves for a correct detection and classification of the occlusion events.

\section{Our improved DP algorithm}

This section describes the proposed matching algorithm. Our improved DP technique produces better occlusion classification and improved disparity maps which, in turn, yield the removal of rendering artefacts in the synthesized virtual images.

Computing matching costs. In order to aid inter-scanline consistency the matching cost $M(l, r)$ is calculated for every pair of pixels along corresponding epipolar lines with a windowed normalised cross-correlation: $M(l, r)=(1-$ $\left.M^{\prime}(l, r)\right) / 2$ where

$$
M^{\prime}(l, r)=\frac{\sum\left(I_{L}-\bar{I}_{L}\right)\left(I_{R}-\bar{I}_{R}\right)}{\sqrt{\sum\left(I_{L}-\bar{I}_{L}\right)^{2} \sum\left(I_{R}-\bar{I}_{R}\right)^{2}}}
$$

is the correlation coefficient. Notice that since $-1 \leq M^{\prime}(l, r) \leq+1 \forall l, r$, then $0 \leq M(l, r) \leq 1 \forall l, r$.

Taller neighborhood windows $($ e.g. $3 \times 7)$ help incorporate inter-scanline information better than square windows. Computing the costs $M(l, r)$ can be performed efficiently by expansion of the above equation and keeping track of sums from one pair of epipolar lines to the next.

Filtering the matching costs. The use of windows for the computation of the $M(l, r)$ costs helps to reduce artefacts in the final disparity and occlusion maps but it is not sufficient for the complete removal of the artefacts. Therefore, in order to obtain cleaner disparity and occlusion maps we first take all the matrices of $M(l, r)$ costs associated to each pair of scanlines (note: not the cumulative cost matrices), stack them up together to create a 3D cost space (fig. 5a), and then apply a two-dimensional Gaussian smoothing filter (parallel to the virtual image plane) to the $3 \mathrm{D}$ cost space. The axis of the Gaussian kernel orthogonal to the left and right scanline axes (denoted a in fig. 5b) is responsible for enforcing inter-scanline consistency of the costs and the orthogonal axis (denoted $\mathbf{a}^{\prime}$ in fig. 5b) produces additional smoothing of sharp corners in the occlusion map.

The output of this process is the new set of $M(l, r)$ costs used as input to our improved DP algorithm, described below.

The five-move model. A major drawback to the standard DP approach is that slanted surfaces (e.g. non frontoparallel walls and table tops) in space must be approximated by a combination of diagonal (matched) and horizontal or vertical (occluded) moves. In such a case the occluded moves do not correspond to real occlusions and therefore, in order to disambiguate between "approximating" occluded moves and real occlusions we augment the basic 3-move model by adding a further pair of horizontal and vertical matched moves, thus defining a 5-label (a.k.a. 5 -move) model. The new model is illustrated in the diagram in fig. 6 (to be compared with fig. 4b).

This improved model produces more consistent classification of occluded and matched pixels. In fact, slanted surfaces are correctly approximated by sequences of matched moves only, and "real" occlusion labels are correctly assigned to pixels visible only in one of the two input views. 


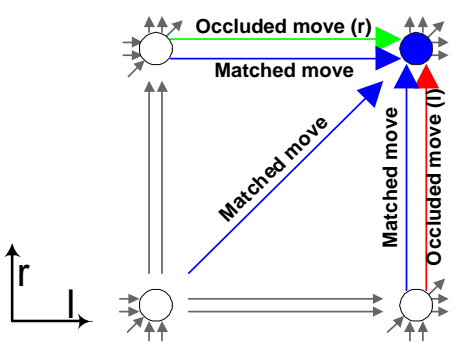

Figure 6: The improved 5-move model for DP. (a) Five different moves are allowed: three matched moves (horizontal, diagonal and vertical), and two occluded moves (horizontal and vertical). Thus, vertical and horizontal moves acquire two possible meanings: (i) for the explicit modeling of occlusion events, and (ii) approximating slanted surfaces.

The three-plane graph for DP. Furthermore, in order to bias towards runs of identical moves we introduce a further extension of the basic DP technique by defining the DP algorithm on three planes of cumulative cost matrices (as opposed to the single plane in [2]): a left-occluded plane L, a matched plane $M$, and a right-occluded plane $R$ (see fig $7 a$ ). As illustrated in fig $7 \mathrm{a}$ in this new model a total of thirteen moves are permitted.

The improved three-plane graph is the basis of our new algorithm for recovering the disparity map. The main advantage of the new model is that it allows us to alter the individual costs of each type of move, independently. For example, biasing the penalty costs against inter-plane moves would tend to keep runs of occluded or non-occluded pixels together, thus reducing most of the inaccuracies in the reconstructed occlusions and disparities. Also, physically impossible moves such as the direct transition between left and right occlusions are prohibited simply by removing certain transitions from the set of allowed ones in the three-plane graph (in fig 7 the top and bottom planes are never directly linked).

At present, the cost $\pi(A \rightarrow B)$ of a generic transition between two planes $A$ and $B$ is manually set, as described below, but further investigation about a possible probabilistic framework is necessary. Moreover, it is reasonable to assume that $\pi(A \rightarrow B)$ is symmetric $^{1}$, i.e. $\pi(A \rightarrow B)=\pi(B \rightarrow A)$ and also that any move involving the left occluded plane has the same cost as a corresponding move involving the right occluded plane. This reduces the total number of penalty parameters to three, and then by setting $\pi(M \rightarrow M)$ to zero in the matched plane we end up with only two parameters: $\alpha$ being the cost of a move within an occluded plane, and $\beta$ being the cost of a move between different planes (fig 7b).

In this new framework the matrices of cumulative costs $\mathrm{C}_{L}, \mathrm{C}_{M}$ and $\mathrm{C}_{R}$ (one for each plane in the graph) are ini-

\footnotetext{
${ }^{1}$ To avoid introducing unjustified asymmetries in the way the image data is treated.
}

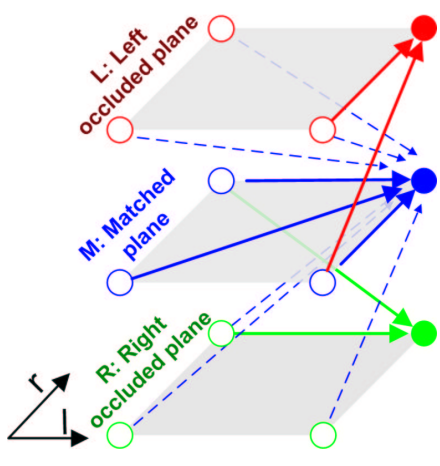

$\mathbf{a}$

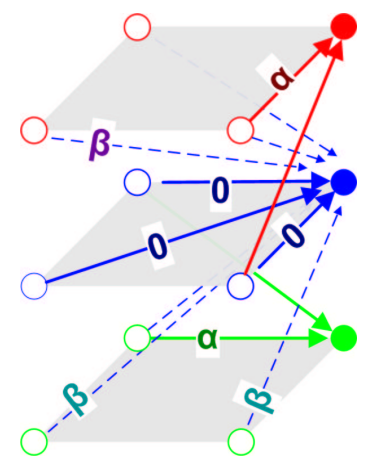

b
Figure 7: The proposed 13-move, 3-plane model for DP. (a) The graph associated to our DP algorithm now lives in three planes to impose constraints between the allowed moves. The allowed moves within planes and between planes are shown by arrows. (b) The permitted moves have been labelled with the associated costs. Some of the labels have been left out for clarity. The entire set of permitted moves and their associated costs is described in the text.

tialised to $\infty$ everywhere except in the right occluded plane, where:

$$
\mathrm{C}_{R}(i, 0)=i \alpha
$$

and then the forward step of the dynamic programming proceeds as follows:

$$
\begin{gathered}
\mathrm{C}_{L}(l, r)=\min \left\{\begin{array}{l}
\mathrm{C}_{L}(l, r-1)+\alpha \\
\mathrm{C}_{M}(l, r-1)+\beta
\end{array}\right. \\
\mathrm{C}_{M}(l, r)=M(l, r)+ \\
\min \begin{cases}\mathrm{C}_{M}(l-1, r) \\
\mathrm{C}_{L}(l-1, r)+\beta \\
\mathrm{C}_{R}(l-1, r)+\beta \\
\mathrm{C}_{M}(l, r-1)+\beta \\
\mathrm{C}_{L}(l, r-1)+\beta \\
\mathrm{C}_{R}(l, r-1)+\beta \\
\mathrm{C}_{M}(l-1, r-1)+\beta \\
\mathrm{C}_{L}(l-1, r-1)+\beta \\
\mathrm{C}_{R}(l-1, r-1)+\beta\end{cases} \\
\mathrm{C}_{R}(l, r)=\min \left\{\begin{array}{l}
\mathrm{C}_{R}(l-1, r)+\alpha \\
\mathrm{C}_{M}(l-1, r)+\beta
\end{array}\right.
\end{gathered}
$$

where $M(l, r)$ is the filtered cost (as described previously) of matching the $l^{\text {th }}$ pixel in the left scanline with the $r^{t h}$ pixel in the right scanline.

The parameters are chosen as follows: $\alpha$ is set to $1 / 2$, a value chosen such that most good matching costs $M(l, r)$ are less than this. The parameter $\beta$ is set to $1.0-\mathrm{a}$ value too low produces spurious isolated matched pixels within occluded regions, with consequent artefacts; while a value too high results in the minimum-cost path rarely leaving the matched plane. 


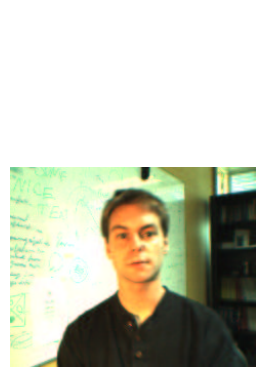

$\mathbf{a}$

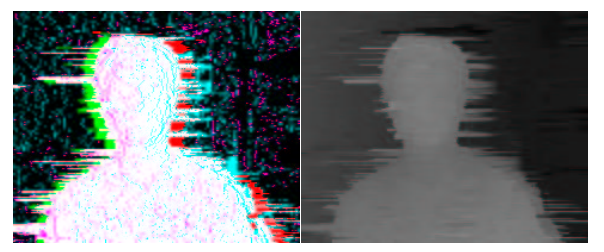

b

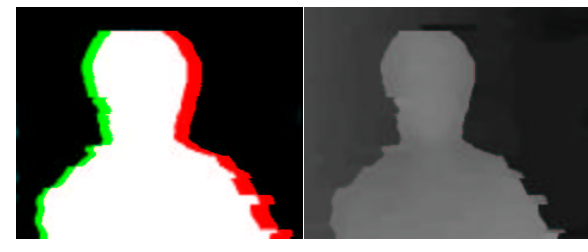

d

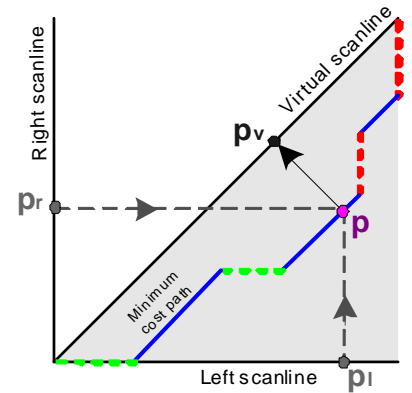

a

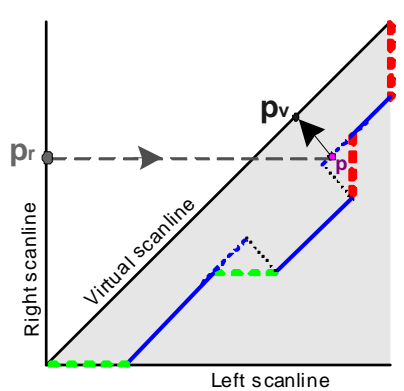

b
Figure 9: Generating the cyclopean view. (a) For matched points: a matched point $\mathbf{p}$ (on blue segments) is projected orthogonally onto its corresponding point $\mathbf{p}_{v}$ on the virtual scanline. The pixel value of the virtual pixel $\mathbf{p}_{v}$ is the average of the corresponding pixels $\mathbf{p}_{l}$ and $\mathbf{p}_{r}$ on left and right images, respectively. (b) For occluded regions: a point $\mathbf{p}$ on the continuation of the background (with same disparity, dashed blue line) is projected orthogonally onto its corresponding point $\mathbf{p}_{v}$ on the virtual scanline. Since we are dealing with a left occlusion then the pixel value for $\mathbf{p}_{v}$ is the same as that of its corresponding point $\mathbf{p}_{r}$ on the right view only. This implements the fronto-parallel occlusion filling directly from the analysis of the graph.

In order to fill the occluded regions, a fronto-parallel assumption is used, i.e. the background is continued at the same depth (dashed lines in fig. 9b). Here, for a left occlusion, the pixel values are taken only from the right image and vice-versa. The disparity value is set accordingly.

The fronto-parallel approximation does not work well if the occlusion regions present isolated matched pixels and therefore, obtaining solid and reliable occlusion regions is of paramount importance. As shown, the use of the proposed thirteen-move, three-plane algorithm with the extra cost-smoothing step produce extremely solid occlusion regions (fig. 8d) and, consequently, visually convincing background propagation into the occlusion regions.

Temporal occlusion filling. Despite the progress obtained in the synthesis of cyclopean views from a stereo pair of still images, when the same algorithm is applied to a sequence of stereo images then small temporal artefacts become visible (e.g. flickering).

To avoid this problem we proceed with a temporal construction of a model of the background that allows us to fill in the regions of missing information at a given time with pixel values which may have been available in previous time instances.

In order to do so the accurately estimated disparity surface is first segmented into foreground and background for each frame by employing the following algorithm: along each scanline in the disparity surface, for each run of occlusions, the disparity at the highest disparity end of the run is histogrammed. The valley in the resulting bi-modal histogram defines the disparity threshold. This is in line 

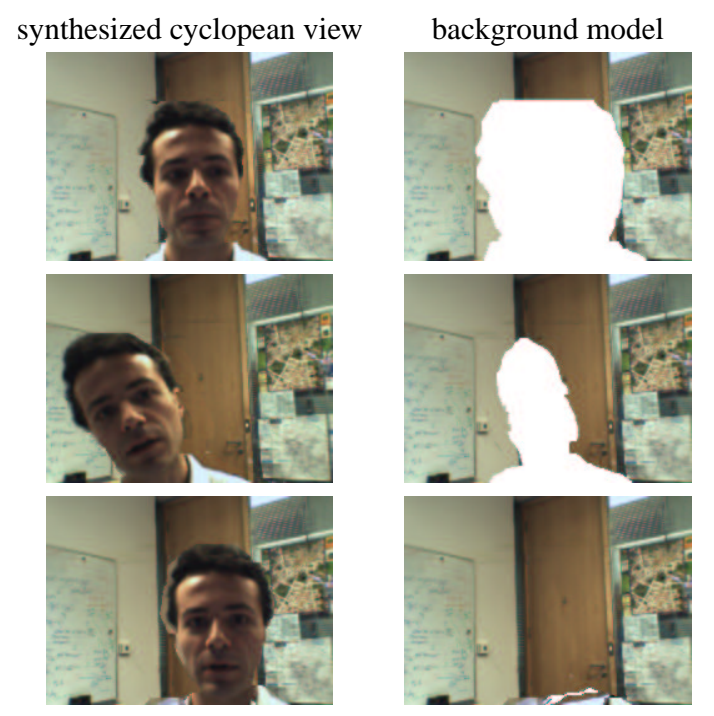

Figure 10: Temporal background generation. (left column) Synthesized cyclopean views for different frames $(0,70$ and 170$)$. More examples of synthesised cyclopean views are provided in the remainder of the paper. (right column) Corresponding cyclopean background models. As new regions of the background are discovered the background model is updated and occluded areas filled in.

with the assumption that almost all runs of occlusions will occur to either side of the head and so the histogram will have a sharp peak where the foreground starts. This turns out to be the case for a large number of sequences and so a foreground threshold disparity can be automatically set. Figure 8d shows also the results of the segmentation (foreground in white and background in black).

Given the segmented foreground and background, new options become available, e.g. to replace the background entirely with a chosen photograph or video, or to dynamically update the background model for use in filling occluded areas in successive frames. The latter is especially useful in the next section which introduces the three-dimensional motion of the virtual camera. In fact, for example, as the virtual camera centre moves away from the baseline of the two input cameras less information is available from individual frames in the occluded regions and temporally acquired information becomes extremely useful.

In the second step of the algorithm a background model is constructed and updated at each time instance. The background model is made of three elements: its disparity map $D_{B}$ in cyclopean coordinates, and the corresponding left and right images $I_{B}^{l}$ and $I_{B}^{r}$, respectively. At each time instance $t$ the background model is updated by the following rule:

$$
\begin{aligned}
D_{B}^{t}(\mathbf{p}) & =\tau D_{B}^{t-1}(\mathbf{p})+(1-\tau) D^{t}(\mathbf{p}) \\
I_{B}^{l^{t}}\left(\mathbf{p}_{l}\right) & =\tau I_{B}^{l^{t-1}}\left(\mathbf{p}_{l}\right)+(1-\tau) I^{t^{t}}\left(\mathbf{p}_{l}\right) \\
I_{B}^{r^{t}}\left(\mathbf{p}_{r}\right) & =\tau I_{B}^{r^{t-1}}\left(\mathbf{p}_{r}\right)+(1-\tau) I^{r^{t}}\left(\mathbf{p}_{r}\right) .
\end{aligned}
$$

where $\mathbf{p}$ is a pixel whose disparity $D(\mathbf{p})$ falls below the

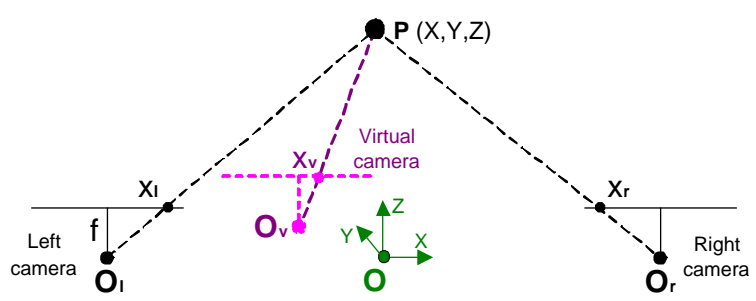

Figure 11: Basic notation for virtual image generation. $\mathbf{O}_{l}, \mathbf{O}_{r}$ and $\mathbf{O}_{v}$ are the optical centres of left, right and virtual cameras respectively. The optical centre of the virtual camera can be placed anywhere in space and the corresponding virtual image is synthesized by our algorithm.

automatically computed foreground threshold $\hat{d}$ (and thus belongs to the background), $\mathbf{p}_{l}$ and $\mathbf{p}_{r}$ are the corresponding positions on left and right input images, respectively. $D_{B}^{t}(\mathbf{p})$ is the disparity of the pixel $\mathbf{p}$ in the current background model at time $t$. The scalar factor $\tau$ represents a decay constant $(0 \leq \tau \leq 1)$ and $I$ indicates intensities.

The update rule (6) applies to all the pixels which belong to the background and are visible, and does not apply to occluded pixels.

The presence of the decay factor $\tau$ (we use $\tau=0.9$ ) in the update rule (6) has the desired effect of temporal smoothing of the output visual data, with the consequent reduction of pixel flicker (instability). This leads to an improved temporal consistency in the reconstructed occluded regions.

Figure 10 illustrates the results of the temporal background filling algorithm.

\section{Simulating the 3D motion of the vir- tual camera}

This section describes a novel, compact technique for rendering virtual views directly from the estimated minimumcost surface, thus negating the need to construct an explicit 3D model of the scene.

Figure 11 shows a plan view of the system with the optical centre of the virtual camera being placed in $\mathbf{O}_{v}$.

A 3D scene point $\mathbf{P}$ is projected on the left and right images into the points $\mathbf{p}_{l}=\left(x_{l}, y_{l}\right)^{\top}$ and $\mathbf{p}_{r}=\left(x_{r}, y_{r}\right)^{\top}$ respectively. Also, $\mathbf{P}$ is projected on the cyclopean camera (with optical centre in $\mathbf{O}_{c}=\mathbf{O}$ ) in the point $\mathbf{p}_{c}=$ $\left(x_{c}, y_{c}\right)^{\top}$ (not shown in the figure) and on the virtual camera (with optical centre $\mathbf{O}_{v}$ in generic position) in the point $\mathbf{p}_{v}=\left(x_{v}, y_{v}\right)^{\top}$. The disparity between the corresponding left and right image points is easily computed as

$$
d=x_{l}-x_{r}=f \frac{B}{Z} .
$$

In the cyclopean camera, by triangle similarity we can compute

$$
x_{c}=f \frac{X}{Z} .
$$




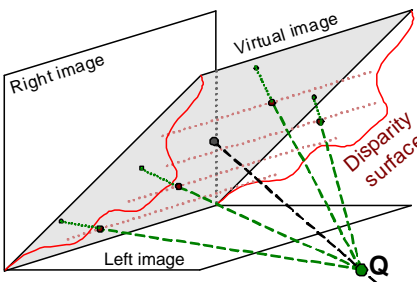

$\mathbf{a}$

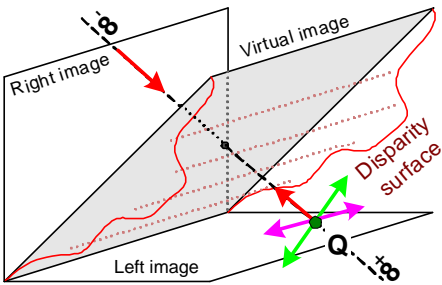

b
- Inwards virtua camera motion
Figure 12: Virtual camera motion. (a) The 3D motion of the virtual camera is achieved by direct projection of points on the minimum-cost surface into the virtual image plane. (b) Moving the centre of projection $\mathbf{Q}$ corresponds to translating the virtual camera. The coloured arrows indicate the mapping between moving the centre of projection $\mathbf{Q}$ in our diagram and the corresponding translations of the virtual camera in the scene.

For a virtual camera with optical center in $\mathbf{O}_{v}=$ $\left(T_{x}, T_{y}, T_{z}\right)^{\top}$ we can write: $\left(X-T_{x}\right): x_{v}=\left(Z-T_{z}\right): f$, from which

$$
x_{v}=f \frac{X-T_{x}}{Z-T_{z}} .
$$

By substituting (7) and (8) into (9) we obtain: $x_{v}=f \frac{x_{c}-d T_{x} / B}{1-d T_{z} /(f B)}$ which, together with the analogous equation for the $y_{v}$ coordinate, can be rewritten in homogeneous coordinates as:

$$
\left(\begin{array}{l}
x_{v} \\
y_{v} \\
w
\end{array}\right)=\left[\begin{array}{cccc}
1 & 0 & -T_{x} / B & 0 \\
0 & 1 & -T_{y} / B & 0 \\
0 & 0 & -T_{z} /(f B) & 1
\end{array}\right]\left(\begin{array}{c}
x_{c} \\
y_{c} \\
d \\
1
\end{array}\right)
$$

Equation (10) represents a projection of 3D points into a plane. It can be proven that (10) corresponds to projecting points of the disparity surface into the corresponding points on the plane of the virtual image (up to a scale, diagonal matrix) as illustrated in fig. 12a.

From (10) the centre of projection $\mathbf{Q}$ is readily computed as the null vector of the projection matrix, thus yielding: $\mathbf{Q}=\left(\begin{array}{cccc}\frac{T_{x}}{B} & \frac{T_{y}}{B} & 1 & \frac{T_{z}}{f B}\end{array}\right)^{\top}$.

Notice that for $T_{z}=0$ the transformation (10) is a parallel projection ( $\mathbf{Q}$ is at infinity). This, in turn means that sidewise (in the $X$ direction) and up/down (in the $Y$ direction) motion of the virtual camera can be simulated by simple projection of points of the min-cost surface onto the virtual image plane via parallel rays.

The inwards/outwards translation of the virtual camera $\left(T_{z} \neq 0\right)$, instead, is achieved by means of a central projection with finite centre of projection $\mathbf{Q}$.

The simple mapping between the motion of the centre of projection $\mathbf{Q}$ and the corresponding translation of the virtual camera is illustrated in $12 \mathrm{~b}$. For instance, inwards camera translation (not zoom) is achieved by moving the centre $\mathrm{Q}$ from $+\infty$ towards the plane of the virtual image.

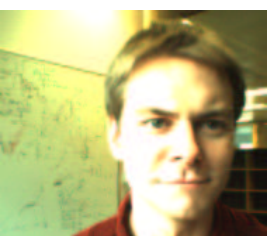

a

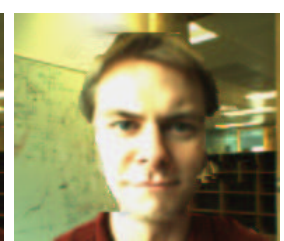

b

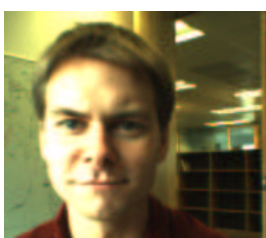

c
Figure 13: Example of gaze correction. (a,c) Input left and right views, respectively; (b) Our algorithm does correct the gaze while eliminating the artefacts. To be compared with fig. 2 .

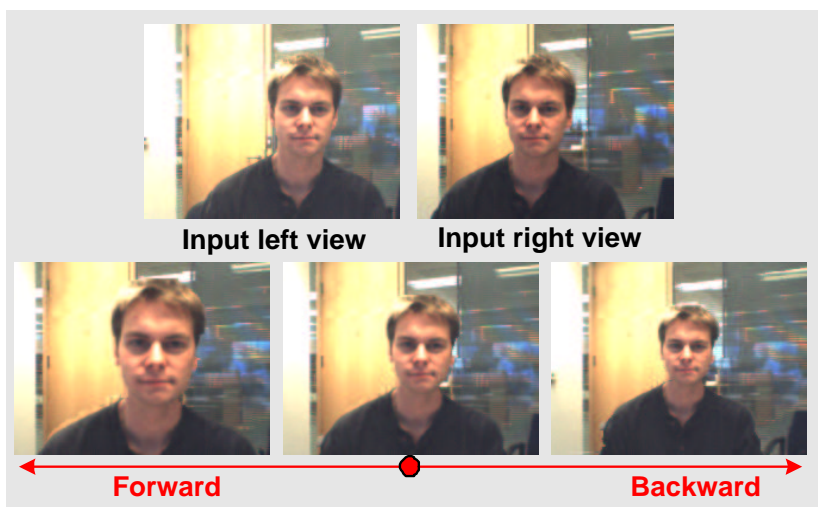

Figure 14: Forward/backward translation of virtual camera. The bottom row shows the synthesized cyclopean views with (left) forward virtual camera translation, (center) cyclopean view, (right) backward virtual camera translation. Notice the parallax effect around the head.

Notice that for $\mathbf{Q}=(-1 / 2,0,1,0)^{\top}$ (i.e. $\mathbf{O}_{v}=$ $\left.(-B / 2,0,0)^{\top}\right)$ the virtual image corresponds to the input left image, for $\mathbf{Q}=(1 / 2,0,1,0)^{\top}$ (i.e. $\mathbf{O}_{v}=$ $\left.(B / 2,0,0)^{\top}\right)$ the virtual image corresponds to the input right image, and for $\mathbf{Q}=(0,0,1,0)^{\top}$ (i.e. $\mathbf{O}_{v}=$ $\left.(0,0,0)^{\top}\right)$ the virtual image corresponds to the half-way cyclopean image.

In order to produce high quality output images inverse mapping and bilinear interpolation techniques are used.

\section{Results}

Generating cyclopean views from still image pairs. Figure 13 shows an example where the input left and right images of fig. 2 have been used to generate the cyclopean view via our algorithm. Notice that the spatial artefacts (streaks in fig. 2b) have been considerably reduced. In the central image the gaze has been corrected.

3D translation of the virtual camera. Figure 14 shows an example of translating the virtual camera towards and away from the viewed scene. This is different from simple zooming or cropping of the output image. In fact, parallax effect may be noticed in the boundary between the head and the background, thus providing the correct threedimensional feeling. 


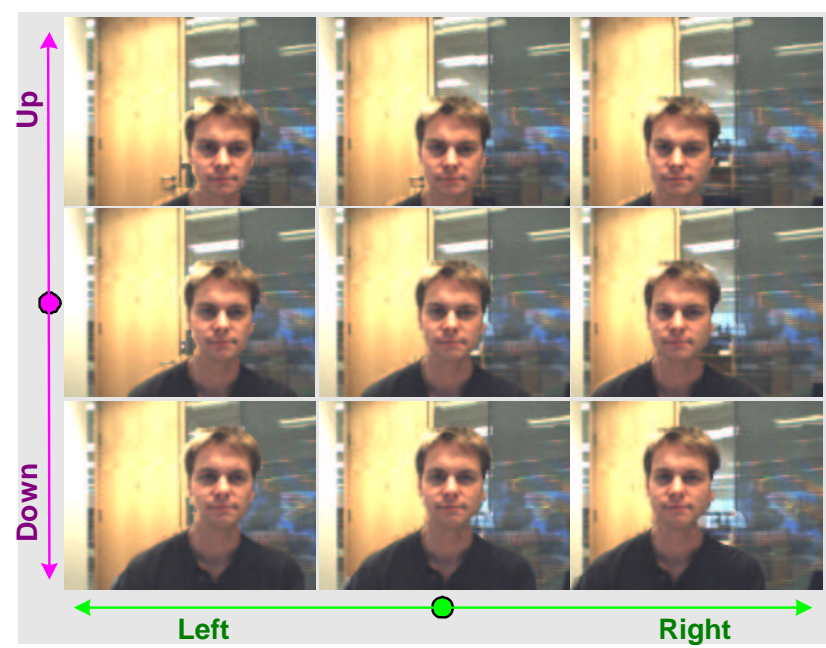

Figure 15: In-plane translation of virtual camera. The left and right input images are the same as in fig. 14. This table shows the synthesized images corresponding to translation of the virtual camera along the $x$ and $y$ axes. Notice the parallax effect around the head. Also, the door frame is reconstructed nicely despite its partial occlusion in the right input view.

Figure 15 shows an example of in-plane translation (along the $X$ and $Y$ directions) of the virtual camera. Notice the relative displacement of the head with respect to the background.

Cyclopean view generation in long sequences. Finally fig. 16 demonstrates the effectiveness of the proposed algorithm for reconstructing cyclopean views of extended temporal sequences. Notice that most of the spatial and temporal artefacts are removed.

\section{Conclusions and Future Work}

We have presented an efficient algorithm for the synthesis and geometric manipulation of high-quality virtual images from a pair of synchronized stereo sequences with large disparities $(0-80$ pixels $)$. In this paper we have focused on one-to-one teleconferencing applications but the techniques described are more general and can be employed in other applications requiring novel view generation and dense stereo.

The newly proposed three-layer graph for dynamic programming, the anisotropic cost filtering and the temporal background model building have been demonstrated effective in the synthesis of novel views with a virtual camera placed in a generic location.

With the current unoptimized implementation these results have been produced at a rate of about a frame every 2 $\sec$ (on $320 \times 240$ images, on a $2.8 G h z$ Pentium).

Finally, for the future development of the work presented in this paper, thorough experimentation with different camera layouts, code optimization for real-time synthesis, and

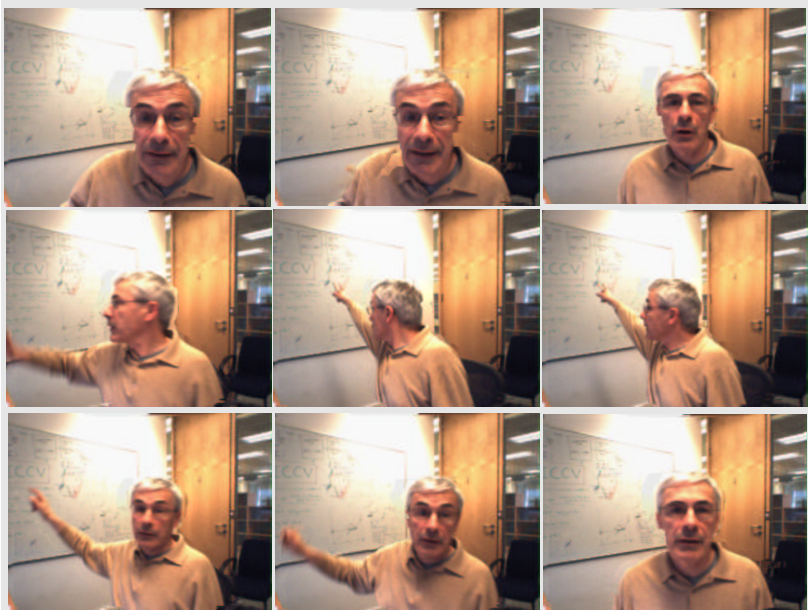

Figure 16: Gaze correction for long sequences. Frames extracted from a reconstructed cyclopean sequence (over $10 \mathrm{sec}$ long).

the generation of standard test sequences for evaluation of the algorithm will be necessary.

Acknowledgements. The authors would like to thank C.Rother, I.Cox, P.Anandan and R.Szeliski for their useful comments and inspiring discussions.

\section{References}

[1] E. Chen and L. Williams. View interpolation for image synthesis. In SIGGRAPH, pages 279-288, 1993.

[2] I. Cox, M. Ott, and J.P. Lewis. Videoconference system using a virtual camera image. US Patent, 5,359,362, 1993.

[3] I.J. Cox, S.L. Hingorani, and S.B. Rao. A maximum likelihood stereo algorithm. Computer vision and image understanding, 63(3):542567, 1996.

[4] J. Gemmell, K. Toyama, C. Zitnick, T. Kang, and S. Seitz. Gaze awareness for video-conferencing: A software approach. IEEE Multimedia, 7(4), 2000.

[5] V. Kolmogorov and R. Zabih. Multi-camera scene reconstruction via graph cuts. In Proc. Europ. Conf. Computer Vision, Copenhagen, Denmark, May 2002.

[6] Y. Ohta and T. Kanade. Stereo by intra- and inter-scan line search using dynamic programming. IEEE Trans. on Pattern Analysis and Machine Intelligence, 7(2):139-154, 1985.

[7] S. Roy and I.J. Cox. A maximum-flow formulation of the n-camera stereo correspondence problem. In Proc. Int. Conf. Computer Vision, pages 492-499, 1998.

[8] D. Scharstein and R. Szeliski. A taxonomy and evaluation of dense two-frame stereo correspondence algorithms. Int. J. Computer Vision, 47(1-3):7-42, 2002.

[9] J. Sun, H. Y. Shum, and N. N. Zheng. Stereo matching using belief propagation. In Proc. Europ. Conf. Computer Vision, Copenhagen, Denmark, May 2002.

[10] T. Vetter. Synthesis of novel views from a single face image. Int. J. Computer Vision, 28(2):103-116, 1998.

[11] R. Yang and Z. Zhang. Eye gaze correction with stereovision for video tele-conferencing. In Proc. Europ. Conf. Computer Vision, volume 2, pages 479-494, Copenhagen, Denmark, May 2002. 